\title{
Tabagismo passivo no Brasil: Resultados da Pesquisa Especial Do Tabagismo, 2008.
}

\author{
Passive smoking in Brazil: \\ Results from the 2008 Special Survey on Smoking
}

Valéria Maria de Azeredo Passos

Luana Giatti ${ }^{2}$

Sandhi Maria Barreto ${ }^{2}$

${ }^{1}$ Departamento de Clínica Médica, Faculdade de Medicina da Universidade Federal de Minas Gerais. Av. Alfredo Balena 190, Bairro Santa Efigênia. 30130-100 Belo Horizonte MG. vpassos@medicina.ufmg.br ${ }^{2}$ Departamento de Medicina Preventiva e Social, Faculdade de Medicina da UFMG,
Abstract The scope of this article was to describe indoor passive smoking, at home and at work, among Brazilians (15+ years) participants of the "Special Research on Smoking", a sub-sample of the PNAD 2008. Non-smokers who reported exposure to indoor household smoking were classified as daily or occasional passive smokers, based on the frequency of exposure. Associations with socio-demographic factors were verified by logistic regression analysis. Among 25,005 participants, prevalence of daily and occasional household exposure were $12.5 \%$ and $21 \%$, respectively. Compared to those not exposed, daily exposure decreased with increasing age, schooling and income. Occasional exposure is not influenced by age, but decreases with increasing schooling and income. Compared to the Southeast Region, daily exposure was lower in the North and Central West and higher in the Northeast. Among those 10,933 with indoor occupations, 55\% of men and 45\% of women reported worksite exposure and 67\% of them also reported household exposure. Prevalence of exposure at work is higher in men, older adults (55+ years) and among those with lower schooling and income and lower among South Region residents. Social inequality in Brazil is seen in greater exposure to passive smoking among those with lower incomes and less education.

Key words Passive smoking, Prevalence, Population-based study, Inequalities
Resumo O objetivo foi descrever prevalência e características sócio-demográficas associadas ao tabagismo passivo no domicílio e no trabalho, em participantes (15+ anos) de amostra populacional da Pesquisa Especial do Tabagismo, sub-amostra da PNAD 2008. O tabagismo passivo é definido como a exposição ao tabaco por não-fumante, em casa, no trabalho ou em outros locais fechados, excluídas ocupações ao ar livre. Associações com características sócio-demográficas foram estimadas por análise de regressão logística. Nos 25.005 não-fumantes, a exposição domiciliar é diária para $12,5 \%$ e ocasional para $21 \%$. À regressão multinomial (referência: não-expostos), a exposição diária diminui com aumento de idade e tanto a exposição diária como a ocasional diminuem com maiores escolaridade e renda. Comparada à Região Sudeste, há menor exposição diária no Norte e no Centro-Oeste, e maior ocasional no Nordeste. Dos 10.933 trabalhadores, $55 \%$ dos homens e $45 \%$ das mulheres relataram exposição no trabalho e $67 \%$ exposição domiciliar adicional. A exposição no trabalho é maior em homens, nos mais velhos (55+anos) e com menor escolaridade e renda; e menor no Sul. A desigualdade social no Brasil se revela também na maior exposição ao tabagismo passivo daqueles com menor escolaridade e renda.

Palavras-chave Tabagismo passivo, Prevalência, Estudo de base populacional, Desigualdade 


\section{Introdução}

Produtos de combustão advindos do cigarro e liberados no ambiente são causa de doenças e de morte prematura. Em todo o mundo, estudos epidemiológicos têm demonstrado forte associação causal entre o tabagismo passivo ambiental e o maior risco de morbimortalidade ${ }^{1}$. Acreditase que a exposição ao tabaco do não fumante que convive com um fumante seja equivalente a $1 \%$ de 20 cigarros fumados ativamente ao $\mathrm{dia}^{2}$.

A presença constante de fumaça do tabaco no ambiente está associada a $25-35 \%$ de aumento de risco de doença arterial coronariana. Apesar da dose de fumo ingerida por fumantes ser 100 vezes superior à ingerida por fumantes passivos, o risco relativo de doença arterial coronariana não é muito diferente, 1,78 para fumantes ativos e 1,31 para fumantes passivos ${ }^{3}$. Morar com um fumante também está associado ao aumento de $20-30 \%$ no risco de desenvolvimento de câncer de pulmão ${ }^{4}$.

Foi demonstrado, em meta-análise reunindo estudos de coorte e ensaios clínicos controlados, aumento de $15 \%$ na mortalidade por doenças cardiovasculares em mulheres com exposição ambiental ao tabaco ${ }^{5}$. Entre crianças, o tabagismo passivo aumenta o risco de tosse, sibilos, admissões hospitalares por doenças respiratórias e de morte súbita; além de retardar o crescimento intrauterino e o desenvolvimento pulmonar ${ }^{6-8}$. O tabagismo passivo, no domicílio ou em ambientes externos, foi também associado ao tabagismo de escolares em inquérito escolar no Brasil ${ }^{9}$.

Os principais locais de exposição passiva ao tabaco são os micro-ambientes representados pelos domicílios, locais de trabalho e locais públicos como restaurantes, lojas e bares. Nos Estados Unidos da América, os adultos passam em média $87 \%$ de seu tempo em prédios, $6 \%$ dentro de veículos e $7 \%$ ao ar-livre ${ }^{4}$.

No Brasil, até o momento, os estudos sobre tabagismo passivo têm focado apenas alguns grupos populacionais de maior risco, tais como crianças ${ }^{6,8,9}$, mulheres grávidas ${ }^{10,11}$ e os portadores de doenças respiratórias ${ }^{12}$.

Pela primeira vez, é possível estimar a exposição ao tabagismo passivo na população adulta urbana brasileira, em decorrência da inserção, em sub-amostra da Pesquisa Nacional por Amostra de Domicílios (PNAD), realizada em 2008, de um complemento especial sobre tabagismo. Este estudo tem por objetivo descrever a prevalência de tabagismo passivo no domicílio e no trabalho em amostra populacional com idade igual ou supe- rior a 15 anos e investigar se esta prevalência varia segundo características sócio-demográficas.

\section{Métodos}

Foram entrevistados 39.425 participantes. Nesse estudo, foram incluídos 25.005 homens e mulheres, que declararam nunca ter fumado na vida, com idade de 15 ou mais anos e que participaram da Pesquisa Especial do Tabagismo (PETab), amostra representativa da população brasileira acima de 15 anos de idade, que utilizou a estrutura amostral da Pesquisa Nacional por Amostra de Domicílios (PNAD), realizada em $2008^{13}$.

A PNAD é um inquérito populacional de âmbito nacional, realizado anualmente pela Fundação Instituto Brasileiro de Geografia e Estatística (IBGE) $)^{13}$. Na PNAD 2008 foram obtidas informações de 391.868 participantes sobre características sócio-demográficas, educação, trabalho, dentre outras. Nesse ano, além da inclusão da PETab, foi introduzido o suplemento específico de saúde já usados em 1998 e 2003.

A PNAD utiliza uma amostra probabilística complexa obtida em três estágios de seleção: unidades primárias - municípios; unidades secundárias - setores censitários; e unidades terciárias - domicílios particulares. No último estágio de seleção da amostra da PNAD, foi selecionado um morador com 15 anos ou mais de idade em cada domicílio por amostra aleatória simples para responder o questionário da PETab. O questionário da PETab foi respondido por 39.435 moradores selecionados, o que implicou em uma modificação na metodologia padrão da PNAD que utiliza informante proxy para obter informações sobre os moradores que não estão presentes no momento da visita do entrevistador ao domicílio ${ }^{14}$.

\section{Variáveis do estudo}

Nessa análise foram consideradas duas variáveis resposta: tabagismo passivo no domicílio e tabagismo passivo no trabalho. Foi definido como tabagismo passivo no domicílio o relato de exposição ambiental à fumaça de tabaco construída a partir das perguntas É permitido fumar em todos os lugares dentro do seu domicílio? e Qual a frequência com que alguém fuma dentro do domicílio? e categorizada em diária, semanal, mensal, menor que mensal e nunca. Para a definição de tabagismo passivo no trabalho, foram excluídas as ocupações ao ar livre e utilizada a pergun- 
ta Existem normas sobre fumar em ambientes fechados onde trabalha? Se a resposta foi uma das opções não sabe, é permitido fumar em qualquer lugar, é permitido fumar em alguns ambientes fechados, não há norma, foi considerado que havia exposição, enquanto que se a resposta foi não é permitido fumar em nenhum ambiente fechado considerou-se ausência de exposição. Na PETab, não foi aferida a frequência da exposição ao tabagismo passivo no trabalho ${ }^{14}$.

Foram incluídas as seguintes características sócio-demográficas: sexo (masculino, feminino), idade em anos (15-24, 25-34, 35-44, 45-54, 5564, 65 e mais), escolaridade em anos completos de estudo (0-3, 4-7, 8-10, 11-14, 15 e mais), renda domiciliar per capita em reais, agrupada em quintis e macro-região de residência (sudeste, sul, nordeste, norte, centro-oeste).

\section{Análise estatística}

Foram também estimadas as prevalências segundo as características sócio-demográficas e as diferenças entre estas, verificadas por meio do teste de qui-quadrado de Pearson. A análise dos fatores associados ao tabagismo passivo no domicilio utilizou a regressão logística multinomial, com a variável dependente, tabagismo passivo no domicílio, agrupada em ausente, diária e ocasional. A categoria de referência para essa análise foi a ausência de exposição. A investigação dos fatores associados ao tabagismo no trabalho foi realizada por regressão logística binária, entre expostos e não expostos. Em ambas as análises, as associações que apresentaram significância estatística com valor de $\mathrm{p}<0,20$ na análise univariada foram incluídas no modelo multivariado. A magnitude das associações foi estimada pelo odds ratio e respectivos intervalos de confiança de 95\%. Considerou-se estatisticamente significativa a associação cujo valor de $\mathrm{p}$ foi menor que $0,05^{15}$.

A análise incorporou pesos para corrigir as diferentes probabilidades de seleção dos participantes. Os percentuais apresentados são, portanto, ponderados. Utilizou-se o programa STATA $10.0^{16}$ que dispõe de procedimentos para análise de inquéritos amostrais complexos e permitem incorporar os pesos distintos das observações que influenciam as estimativas pontuais de parâmetros da população total e os efeitos do plano amostral sobre as estimativas de variância e desvio-padrão.

\section{Resultados}

\section{Tabagismo passivo no domicílio}

Dos 25.005 participantes não fumantes do estudo sobre exposição domiciliar, 57,7\% eram mulheres, 53,8\% tinham até 34 anos, cerca de 60\% tinham até 10 anos de escolaridade e relataram renda domiciliar per capita de até R\$ 500,00 (salário mínimo vigente de $\mathrm{R} \$ 415,00)$. A maioria da população entrevistada residia na Região Sudeste (44,3\%).

A exposição domiciliar foi diária para $12,5 \%$, semanal para 3,6\% e mensal ou menos para $17,4 \%$ dos participantes do estudo. Entre os expostos, 50,6\% eram homens, $80 \%$ tinham até 44 anos, $73 \%$ relataram renda mensal pessoal de até $\mathrm{R} \$ 900,00$ e $59 \%$ tinham pelo menos 11 anos de escolaridade. Como a exposição domiciliar semanal foi pequena, optou-se por analisar diferenças na exposição domiciliar agrupando as categorias em diária, ocasional ou ausente. $\mathrm{Na}$ análise univariada, não foi encontrada associação entre a exposição domiciliar e o sexo, mas foram observadas diferenças significantes na exposição com relação à idade, escolaridade, renda e região de residência (tabela 1 ).

A análise multinomial, tendo como referência a população não exposta, mostrou que a exposição diária diminuiu significativamente com o aumento da idade, da escolaridade e da renda. Já a exposição ocasional não sofreu influência da idade, mas diminuiu com maiores escolaridade $\mathrm{e}$ renda. Observou-se também menor chance de residir nas regiões Norte e Centro-Oeste do país quando comparadas à região Sudeste entre os indivíduos que relataram exposição diária. Entre os que relataram exposição ocasional, a chance de residir na região Nordeste foi superior ao da região Sudeste (tabela 2 ).

\section{Tabagismo no passivo no trabalho}

Do total de 10.933 pessoas que relataram trabalhar em ocupações em ambientes fechados, $50,6 \%$ era do sexo masculino, a grande maioria jovem (80\% tinha até 44 anos de idade), com escolaridade igual ou superior a 11 anos de estudo (59\%) e 73,5\% informaram renda domiciliar per capita de até R\$900,00. A exposição ambiental ao tabaco no domicílio foi relatada por $67 \%$ desses trabalhadores.

Quando observamos a proporção da população exposta ao tabagismo passivo no trabalho, percebemos que é maior em homens, nos adultos mais velhos (55-64 anos) e nos idosos 
Tabela 1. Distribuição de frequência de exposição ao tabagismo passivo no domicílio, por características sócio-demográficas e percepção da saúde dos participantes da Pesquisa Especial do Tabagismo, PNAD 2008.

\begin{tabular}{|c|c|c|c|}
\hline \multirow[b]{2}{*}{ Características } & \multicolumn{3}{|c|}{ Frequência de exposição } \\
\hline & Nunca & Ocasional & Diária \\
\hline \multicolumn{4}{|l|}{ Sexo } \\
\hline Masculino & 66,6 & 21,4 & 12,0 \\
\hline Feminino & 66,2 & 20,9 & 12,9 \\
\hline Faixa etária (anos) & $\chi^{2}=6,94$ & \multicolumn{2}{|c|}{ valor de $\mathrm{p}=0,22$} \\
\hline $15-24$ & 60,3 & 19,6 & 20,1 \\
\hline $25-34$ & 67,9 & 22,2 & 9,9 \\
\hline $35-44$ & 69,6 & 22,4 & 8,0 \\
\hline $45-54$ & 70,2 & 20,6 & 9,2 \\
\hline $55-64$ & 69,1 & 20,4 & 10,5 \\
\hline $65+$ & 68,9 & 21,8 & 9,3 \\
\hline \multicolumn{4}{|l|}{ Escolaridade } \\
\hline $0-3$ & 59,5 & 25,0 & 15,5 \\
\hline $4-7$ & 63,5 & 22,5 & 14,0 \\
\hline $8-10$ & 64,9 & 19,9 & 15,2 \\
\hline $11-15$ & 70,8 & 19,5 & 9,7 \\
\hline $15+$ & 73,3 & 19,4 & 7,3 \\
\hline \multicolumn{4}{|c|}{$\begin{array}{l}\text { Renda domiciliar } \\
\text { per capita (reais) }\end{array}$} \\
\hline$\leq 185$ & 57,4 & 24,9 & 17,7 \\
\hline$>185$ a 325 & 63,6 & 21,3 & 15,1 \\
\hline$>325$ a 500 & 67,9 & 20,8 & 11,3 \\
\hline$>500$ a 900 & 69,7 & 19,8 & 10,5 \\
\hline$>900$ & 72,4 & 19,3 & 8,2 \\
\hline \multicolumn{4}{|l|}{ Região do país } \\
\hline Sudeste & 68,2 & 19,9 & 11,9 \\
\hline Sul & 66,3 & 21,8 & 11,8 \\
\hline Nordeste & 61,9 & 23,2 & 14,9 \\
\hline Norte & 69,1 & 19,5 & 11,4 \\
\hline \multirow[t]{2}{*}{ Centro-oeste } & 69,1 & 20,5 & 10,4 \\
\hline & $\chi^{2}=98,6$ & valor de $\mathrm{p}=$ &, 00001 \\
\hline
\end{tabular}

(65+ anos), naqueles com menor escolaridade (0-7 anos) e nos indivíduos com renda per capita de até $\mathrm{R} \$ 500,00$ (tabela 3).

A análise logística mostrou menor chance de exposição em mulheres ( $\mathrm{OR}=0,79$, IC95\% 0,70$0,89)$, naqueles com maior escolaridade e maior renda, e nos residentes da região Sul $(\mathrm{OR}=0,86$, IC95\% 0,76-0,99). Houve maior chance de exposição em idosos (OR=2,08, IC95\% 1,29-3,33) (tabela 3 ).
Tabela 2. Associação entre frequência de exposição domiciliar ao tabagismo passivo e características sócio-demográficas no Brasil. Pesquisa Especial do Tabagismo, PNAD 2008 (categoria de referência = sem exposição).

\begin{tabular}{|c|c|c|}
\hline \multirow[b]{2}{*}{ Características } & \multicolumn{2}{|c|}{ Frequência de exposição * } \\
\hline & Ocasional & Diária \\
\hline \multicolumn{3}{|l|}{ Faixa etária (anos) } \\
\hline $15-24$ & 1,00 & 1,00 \\
\hline $25-34$ & $1,02(0,91-1,13)$ & $0,45(0,39-0,51)$ \\
\hline $35-44$ & $0,97(0,86-1,09)$ & $0,33(0,28-0,38)$ \\
\hline $45-54$ & $0,85(0,75-0,99)$ & $0,36(0,30-0,44)$ \\
\hline $55-64$ & $0,84(0,71-0,98)$ & $0,40(0,32-0,50)$ \\
\hline $65+$ & $0,88(0,74-1,03)$ & $0,31(0,25-0,40)$ \\
\hline \multicolumn{3}{|l|}{ Escolaridade (anos) } \\
\hline $0-3$ & 1,00 & 1,00 \\
\hline $4-7$ & $0,83(0,73-0,93)$ & $0,67(0,57-0,78)$ \\
\hline $8-10$ & $0,72(0,63-0,83)$ & $0,59(0,50-0,70)$ \\
\hline $11-15$ & $0,68(0,59-0,77)$ & $0,44(0,37-0,53)$ \\
\hline $15+$ & $0,68(0,56-0,82)$ & $0,47(0,36-0,64)$ \\
\hline \multicolumn{3}{|l|}{$\begin{array}{l}\text { Renda domiciliar } \\
\text { per capita (reais) }\end{array}$} \\
\hline$\leq 185$ & 1,00 & 1,00 \\
\hline$>185$ a 325 & $0,83(0,73-0,93)$ & $0,87(0,756-1,01)$ \\
\hline$>325$ a 500 & $0,78(0,69-0,88)$ & $0,68(0,57-0,80)$ \\
\hline$>500$ a 900 & $0,75(0,66-0,86)$ & $0,65(0,55-0,78)$ \\
\hline$>900$ & $0,75(0,65-0,86)$ & $0,58(0,46-0,71)$ \\
\hline \multicolumn{3}{|l|}{ Região do país } \\
\hline Sudeste & 1,00 & 1,00 \\
\hline Sul & $1,12(1,00-1,25)$ & $1,03(0,89-1,21)$ \\
\hline Nordeste & $1,11(1,06-1,23)$ & $0,98(0,86-1,12)$ \\
\hline Norte & $0,89(0,70-1,02)$ & $0,73(0,60-0,88)$ \\
\hline Centro-oeste & $0,99(0,88-1,11)$ & $0,78(0,66-0,95)$ \\
\hline
\end{tabular}

"regressão logítica multinomial, ajuste por todas as variáveis.

\section{Discussão}

A exposição domiciliar ao tabaco no Brasil, em torno de $33 \%$, em 2008, pode ser considerada bastante alta. Prevalência que se repete no ambiente de trabalho, onde encontramos 55\% de homens e $45 \%$ de mulheres expostos. Na Coreia do Sul, em 2007, resultados de inquérito telefônico em Seul revelaram prevalência de $26 \%$ e $25 \%$ para tabagismo passivo em casa e no trabalho, respectivamente ${ }^{17}$. Uma abrangente revisão de estudos sobre tabagismo passivo nos Estados Unidos da América (EUA), com inquéritos em adultos não fumantes ou ex-fumantes, já mostrava prevalências menores, entre 17 e $24 \%$, com queda em torno de 77\% entre 1988-1991 e 2001$2002^{18}$. Também nos EUA, a percentagem de trabalhadores em ambientes livres do tabaco cresceu de 35\% em 1990 para 93\% em 1999, o que 
Tabela 3. Associação entre tabagismo passivo no trabalho e características sócio-demográficas no Brasil. Pesquisa Especial do Tabagismo, PNAD 2008 (categoria referência=não-exposição).

\begin{tabular}{|c|c|c|c|c|}
\hline Características & $\begin{array}{c}\text { Amostra }(\%) \\
n=10.933\end{array}$ & $\begin{array}{c}\text { Prevalência de exposição } \\
\text { no trabalho }(\%)\end{array}$ & $\begin{array}{l}\text { OR }(\text { IC95\%) } \\
\text { Não ajustado }\end{array}$ & $\begin{array}{l}\text { OR }(\text { IC95\%) } \\
\text { Ajustado* }\end{array}$ \\
\hline \multicolumn{5}{|l|}{ Sexo } \\
\hline Masculino & 50,6 & 55,4 & 1,00 & 1,00 \\
\hline Feminino & 49,4 & 44,6 & $0,77(0,69-0,86))$ & $0,79(0,70-0,89)$ \\
\hline & \multicolumn{4}{|c|}{$\chi^{2}=32,75$ valor de $p=0,00001$} \\
\hline \multicolumn{5}{|l|}{ Faixa etária (anos) } \\
\hline $15-24$ & 25,1 & 23,0 & 1,00 & 1,00 \\
\hline $25-34$ & 31,7 & 30,4 & $1,06(0,91-1,24)$ & $1,13(0.96-1,32)$ \\
\hline $35-44$ & 23,7 & 25,0 & $1,20(1,02-1,41)$ & $1,21(1,01-1,43)$ \\
\hline $45-54$ & 13,5 & 14,2 & $1,20(0,98-1,46)$ & $1,18(0,95-1,47)$ \\
\hline $55-64$ & 4,8 & 5,4 & $1,33(1,02-1,73)$ & $1,21(0,91-1,63)$ \\
\hline $65+$ & 1,2 & 2,0 & $2,44(1.61-3,70)$ & $2,08(1,29-3,33)$ \\
\hline & \multicolumn{4}{|c|}{$\chi^{2}=34,6$ valor de $\mathrm{p}=0,0005$} \\
\hline \multicolumn{5}{|l|}{ Escolaridade (anos) } \\
\hline $0-3$ & 6,3 & 10,5 & 1,00 & 1,00 \\
\hline $4-7$ & 16,5 & 25,3 & $0,88(0,71-1,09)$ & $0,96(0,76-1,20)$ \\
\hline $8-10$ & 18,0 & 13,2 & $0,51(0,41-0,64)$ & $0,63(0,49-0,79)$ \\
\hline $11-15$ & 44,2 & 36,3 & $0,37(0,30-0,45)$ & $0,48(0,38-0,61)$ \\
\hline $15+$ & 15,0 & 8,7 & $0,24(0,19-0,31)$ & $0,34(0,26-0,46)$ \\
\hline $\begin{array}{l}\text { Renda domiciliar per } \\
\text { capita (reais) }\end{array}$ & \multicolumn{4}{|c|}{$\chi^{2}=408,0$ valor de $\mathrm{p}=0,00001$} \\
\hline$\leq 185$ & 8,8 & 13,3 & 1,00 & 1,00 \\
\hline$>185$ a 325 & 17,1 & 19,9 & $0,66(0,54-0,81)$ & $0,72(0,59-0,89)$ \\
\hline$>325$ a 500 & 21,2 & 24,0 & $0,63(0,52-0,77)$ & $0,75(0,61-0,93)$ \\
\hline$>500$ a 900 & 26,5 & 23,2 & $0,45(0,37-0,55)$ & $0,59(0,47-0,72)$ \\
\hline \multirow[t]{2}{*}{$>900$} & 26,4 & 19,6 & $0,37(0,30-0,45)$ & $0,56(0,44-0,71)$ \\
\hline & \multicolumn{4}{|c|}{$\chi^{2}=188,8$ valor de $\mathrm{p}=0,00001$} \\
\hline \multicolumn{5}{|l|}{ Região do país } \\
\hline Sudeste & 49,7 & 53,2 & 1,00 & 1,00 \\
\hline Sul & 16,2 & 12,2 & $0,64(0,54-0,74)$ & $0,86(0,54-0,75)$ \\
\hline Nordeste & 20,2 & 21,6 & $1,00(0,87-1,14)$ & $0,81(0,69-0,94)$ \\
\hline Norte & 6,7 & 6,5 & $0,88(0,74-1,07)$ & $0,77(0,64-0,94)$ \\
\hline Centro-oeste & 7,2 & 6,5 & $0,81(0,68-0,96)$ & $0,73(0,61-0,87)$ \\
\hline & \multicolumn{4}{|c|}{$\chi^{2}=49,2$ valor de $\mathrm{p}=0,00001$} \\
\hline
\end{tabular}

* regressão logística, ajuste por todas as variáveis.

configura prevalência cinco vezes menor naquele país que no Brasil ${ }^{18}$.

As características sócio-demográficas da população exposta ao tabaco, tanto no domicílio como no trabalho, revelam mais uma vez as desigualdades sociais do Brasil, com maior exposição e risco naqueles com menor escolaridade e renda. Em 2005, a escolaridade também foi inversamente associada ao tabagismo passivo domiciliar em inquérito nacional no Camboja ( $\mathrm{OR}=0,51 \%$, IC-0,27-0,96 para 10 anos versus até 5 anos de escolaridade) ${ }^{19}$. A associação inversa entre exposição ao tabaco nos domicílios e escolaridade e renda também é encontrada para o tabagismo ativo, cada vez mais um problema 
sar que a magnitudes da exposição ao tabaco é dependente de vários fatores, tais como o número de fumantes ativos, o tamanho do ambiente, o tempo de exposição, a frequência de troca de ar no ambiente, etc. como as mulheres tendem a ficar por mais tempo no domicílio, a exposição passiva ao tabagismo pode apresentar riscos específicos à sua saúde reprodutiva. No ambiente de trabalho, as mulheres são menos afetadas que os homens, o que provavelmente espelha diferenças nas ocupações exercidas e, também, menor frequência de colegas de trabalho fumantes.

As ações de saúde pública para eliminar a exposição ao tabagismo ambiental são responsabilidades do Estado, que assim está protegendo o cidadão dos males que outros podem fazer contra ele ${ }^{22}$. Uma das explicações para as menores taxas de exposição nos EUA pode se dever ao fato de que, desde a década de 70, já são aplicadas leis de banimento do cigarro em locais públi$\cos ^{18}$, iniciativa que foi adotada pelo Brasil mais de 20 anos depois (Quadro 1) ${ }^{23}$.

No Brasil, medidas antitabagismo passivo são mais recentes e de menor impacto que aquelas generalistas, o que pode ser uma das razões para sua alta prevalência quando o país apresenta queda no consumo de tabaco ${ }^{20}$. Outros estudos são necessários para a compreensão deste fenômeno.

Medidas de redução de fumaça ambiental têm se mostrado eficazes na redução de riscos à saúde. O número de admissões hospitalares por infarto agudo do miocárdio diminuiu após a implementação de medidas restritivas ao fumar em ambientes públicos e de trabalho ${ }^{17}$. A promoção de ambientes livres de tabaco está entre as seis medidas indicadas pela OMS como prioritárias para adoção nos países do mundo para conter a expansão do tabagismo e suas graves consequências para a saúde ${ }^{24}$.

No Brasil, já vigoram diversas leis relacionadas à proibição do fumo em locais públicos e fechados no país (Quadro 1) 23. Em alguns lugares, como aviões, bancos, veículos de transporte e repartições públicas, elas são respeitadas; porém, em outros, ainda há tolerância. A Lei Federal 9.294/96 restringe o uso de tabaco, mas permite a existência de áreas designadas para fumar, os fumódromos, desde que utilizadas exclusivamente para este fim, com isolamento e arejamento convenientes. Estudos consistentes já demonstram a pouca efetividade da separação de fumantes e não-fumantes em um mesmo ambiente ${ }^{20}$. Recentemente, surgiram leis estaduais proibindo o fumo em bares e restaurantes ${ }^{25}$.
A implementação de políticas de ambientes fechados livres de fumo é hoje uma tendência mundial, fundamentada no conhecimento inequívoco dos males provocados pela exposição aos componentes tóxicos da fumaça de tabaco. No Brasil, pesquisas têm demonstrado com grande apoio popular ao banimento do fumo em ambientes fechados ${ }^{26,27}$.

A região Sudeste, a mais populosa e industrializada, é também aquela onde a prevalência da exposição ao tabaco no trabalho é a maior do país, sendo que sua prevalência em exposição domiciliar é maior que nas regiões Norte e CentroOeste. Medidas de saúde pública distintas devem considerar as especificidades dos diferentes contextos onde pode ocorrer a exposição passiva. No ambiente de trabalho, uma maior fiscalização e o respeito à Lei Federal 9.294/96 pode contribuir objetivamente para a proteção da saúde do trabalhador ${ }^{20}$. No ambiente domiciliar, a exposição está ligada a normas culturais e sociais, por isso precisam ser fortalecidas medidas educativas, que difundam conhecimentos e práticas, envolvendo não apenas o setor saúde, mas também a educação e os meios de comunicação. A ampliação dos ambientes públicos livres de tabaco concorre para a disseminação de redução do tabagismo no domicílio e em ambientes fechados.

Estudos do impacto sobre a saúde, do tabagismo passivo entre adultos, enfrentam desafios metodológicos importantes, tais como separar o efeito do tabagismo passivo de outros fatores de risco a que esta população está exposta. O presente estudo caracteriza a dimensão populacional do tabagismo passivo, sem a pretensão de estudar seu impacto sobre a saúde de toda a população estudada, mas com a intenção de alertar para a magnitude social do problema e fortalecer as iniciativas públicas direcionadas para a redução desse mal em nossa sociedade.

\section{Colaboradores}

VMA Passos, L Giatti e SM Barreto trabalharam na concepção, delineamento, análise e redação do artigo.

\section{Agradecimentos}

SM Barreto e VMA Passos são bolsistas de produtividade em pesquisa do CNPq. L Giatti é bolsista PRODOC- CAPES. 
Quadro 1. Sumário das medidas legislativas para estabelecimento de ambientes livres da exposição ao fumo no Brasil.

\begin{tabular}{|c|c|c|}
\hline Ano & Legislação & Descrição \\
\hline \multicolumn{3}{|c|}{ Proteção contra os riscos da exposição à poluição tabagística ambiental } \\
\hline 1988 & $\begin{array}{l}\text { Portaria Interministerial } \\
\text { no } 3257\end{array}$ & $\begin{array}{l}\text { Recomenda medidas restritivas ao fumo no ambiente de } \\
\text { trabalho }\end{array}$ \\
\hline 1996 & Lei no 9.294 & $\begin{array}{l}\text { Proíbe o uso de qualquer produto fumígeno derivado do } \\
\text { tabaco em recinto coletivo, privado ou público, tais como } \\
\text { repartições públicas, hospitais, salas de aula, ambientes de } \\
\text { trabalho, teatros e cinema. Permite fumódromos. }\end{array}$ \\
\hline 1996 & Decreto no 2018 & $\begin{array}{l}\text { Regulamenta a Lei 9294/96, definindo os conceitos de } \\
\text { "recinto coletivo" e "área devidamente isolada e destinada } \\
\text { exclusivamente ao tabagismo". }\end{array}$ \\
\hline 2002 & $\begin{array}{l}\text { Portaria Interministerial } \\
\text { no }^{\circ} 1498\end{array}$ & $\begin{array}{l}\text { Recomenda às instituições de saúde e de ensino programas } \\
\text { de ambientes livres da exposição ambiental ao tabaco. }\end{array}$ \\
\hline 2006 & $\begin{array}{l}\text { Portaria do Ministério da } \\
\text { Saúde no } 300\end{array}$ & $\begin{array}{l}\text { Proíbe fumar em todas as dependências do Ministério da } \\
\text { Saúde e institui programa "MS livre do Tabaco" }\end{array}$ \\
\hline 2006 & Portaria da Anvisa no 528 & \\
\hline \multicolumn{3}{|c|}{ Ações de conscientização da população } \\
\hline 1986 & Lei no 7488 & Cria "Dia Nacional de Combate ao Fumo". \\
\hline 1988 & $\begin{array}{l}\text { Portaria Interministerial } \\
\text { no } 3257\end{array}$ & $\begin{array}{l}\text { Confere certificados de honra ao mérito às empresas que se } \\
\text { destacarem em campanhas para o controle de tabagismo. }\end{array}$ \\
\hline 2001 & $\begin{array}{l}\text { Medida Provisória } \\
\text { no } 2.190-34\end{array}$ & $\begin{array}{l}\text { Altera lei no 9294/96, determinando que o material de } \\
\text { propaganda e as embalagens de derivados de tabaco, exceto } \\
\text { as destinadas à exportação, contenham advertências } \\
\text { acompanhadas de imagens que ilustrem seu sentido. }\end{array}$ \\
\hline 2002 & $\begin{array}{l}\text { Portaria Interministerial } \\
\text { n }^{\circ} 1498\end{array}$ & $\begin{array}{l}\text { Confere certificados de honra ao mérito às instituições de } \\
\text { saúde e de ensino programas que se destacarem em } \\
\text { campanhas para o controle do tabagismo. }\end{array}$ \\
\hline 2003 & $\begin{array}{l}\text { Resolução da Anvisa } \\
\text { no } 335\end{array}$ & $\begin{array}{l}\text { Determina inserções de frases alusivas à proibição da venda a } \\
\text { menor de } 18 \text { anos e à existência de } 4700 \text { substâncias tóxicas } \\
\text { e nicotina, que causam dependência física e psíquica. }\end{array}$ \\
\hline 2004 & $\begin{array}{l}\text { Portaria Interministerial } \\
\text { n }^{\circ} 1034\end{array}$ & $\begin{array}{l}\text { Institui grupo de trabalho, no âmbito da Secretaria de } \\
\text { Educação à Distância, para promoção do tema "controle do } \\
\text { tabagismo" no recurso didático do ensino á distância, } \\
\text { promovido pelo Programa TV Escola. }\end{array}$ \\
\hline 2007 & $\begin{array}{l}\text { Resolução da Anvisa } \\
\text { no } 10\end{array}$ & $\begin{array}{l}\text { Substitui logo e telefone do serviço "Disque pare de Fumar" } \\
\text { pelo serviço "Disque Saúde" }\end{array}$ \\
\hline
\end{tabular}

Fonte: Legislação Federal Vigente sobre Tabaco no Brasil ${ }^{23}$. 


\section{Referências}

1. Faught BE, Flouris AD, Cairney J. Epidemiological evidence associating secondhand smoke exposure with cardiovascular disease. Inflamm Allergy Drug Targets 2009; 8(5):321-327.

2. Doll R, Crofton J. Tobacco and health. Br Med Bull 1996; 52:22-34.

3. Barnoya J, Stanton AG. Cardiovascular effects of secondhand smoke. Nearly as large as smoking. Circulation 2005; 111:2684-2698.

4. Kepleis NE, Nelson WC, Ott WR, Robinson JP, Tsang AM, Switzer P, Behar JV, Hern SC, Engelmann WH. The national Human Activity Pattern Survey (NHAPS): a resource for assessing exposure to environmental pollutants. J Expo Anal Environ Epidemiol 2001; 11:231-252.

5. Kaur S, Cohen A, Dolor R, Coffman CJ, Bastian LA. The impact of environmental tobacco smoke on women's risk of dying from heart disease: a metaanalysis. J Women Health 2004; 13(8):888-897.

6. Gonçalves-Silva RMV, Valente JG, Lemos-Santos MGF, Sichieri R. Tabagismo no domicílio e doença respiratória em crianças menores de cinco anos. Cad Saude Publica 2006; 22(3):579-586.

7. Moshammer H, Hoek G, Luttmann-Gibson H, Neuberger MA, Antova T, Gehring U, Hruba F, Pattenden S, Rudnai P, Slachtova H, Zlotkowska R, Fletcher T. Parental smoking and lung function in children. An international study. Am J Respir Crit Care Med 2006; 173:1255-1263.

8. Carvalho LMT, Pereira EDB. Morbidade respiratória em crianças fumantes passivas. J Bras Pneumo 2002; 28(1):8-14.

9. Hallal AL, Gottlieb SL, Almeida LM, Casado L. Prevalence and risk factors associated with smoking among school children, Southern Brazil. Rev Saude Publica 2009; 43(5):779-788.

10. Horta BL, Victora CG, Menezes AM, Halpern R, Barros FC. Low birthweight, preterm births and intrauterine growth retardation in relation to maternal smoking. Paediatric Perinat Epidemiol 1997; 11(2):140-151.

11. Nakamura MU, Alexandre SM, Santos JFK, Souza E; Sass N, Beck APA, Trayna, E, Andrade CMA, Barroso T, Kulay Júnior L. Repercussões obstétricas e perinatais do tabagismo (ativo e/ou passivo) na gravidez. São Paulo Med J 2004; 122(3):94-98.

12. Vork K, Broadwin R; Blaisdell R. Asma na infância por exposição ao tabagismo passivo: compreensão a partir de uma meta-regressão Cien Saude Colet 2008; 13(4):1313-1325.

13. Instituto Brasileiro de Geografia e Estatística (IBGE). Pesquisa Nacional por Amostra de Domicílios 2008. [acessado 2010 ago]. Disponível em: http://www.ibge. gov.br/home/presidencia/noticias/noticia_visualiza. php?id_noticia $=1455 \&$ id_pagina

14. Freitas MPS, Lila MF, Antonaci GA, Albieri S. Tamanho de amostra para a pesquisa especial sobre tabagismo PETab. [artigo na internet] [acessado 2010 ago]. Disponível em: http://www.ime.unicamp.br/ sinape/sites/default/files/SINAPE $\% 202010 \% 20$ \%20PETab\%20-\%20Sonia\%20Albieri.pdf

15. Hosmer DW, Lemenshow S. Applied logistic regression $2^{\text {nd }}$ ed. USA: John Wily \& Sons Inc.; 2000.
16. Stata Corporation LP: Statistic/Data Analysis Software (Stata) 9.2. StataCorporation LP, College Station, Texas, USA 2007.

17. Hughes SC, Corcos IA, Hofstetter CR, Hovell MF, Seo DC, Irvin VL, Park HR, Paik HY. Secondhand smoke exposure among nonsmoking adults in Seoul, Korea. Asian Pac J Cancer Prev 2008; 9(2):247-252.

18. US Department of Health and Human Services. The Health consequences of involuntary exposure to tobacco smoke: a report of the Surgeon General. Atlanta, GA: The Department 2006. [acessado 2010 jul]. Disponível em: www.surgeongeneral.gov/library/ secondhandsmoke/.

19. Rudatsikira EM, Knutsen SF, Job JS, Singh PN, Yel D, Montgomery SB, Petersen FF, Ferry LH. Exposure to environmental tobacco smoke in the nonsmoking population of Cambodia. Am J Prev Med 2008; 34(1): 69-73.

20. Cavalcante TM. O controle do tabagismo no Brasil: avanços e desafios. Rev Psiq Clin 2005; 32(5):283300.

21. Wunsch-Filho V, Mirra AP, López RSM, Antunes LF. Tabagismo e câncer no Brasil: evidências e perspectivas. Rev Bras Epidemiol 2010; 13(2):175-187.

22. Oliveira MVC, Sales MPU. Tabagismo passivo. $J$ Bras Pneumo 2004; 30(Supl. 2):S65-S72.

23. Instituto Nacional do Câncer. Legislação Federal Vigente sobre Tabaco no Brasil. [acessado 2010 ago]. Disponível em: http://www.inca.gov.br/tabagismo/ frameset.asp?item=economia\&link=leisfederais.pdf

24. WHO Report on the Global Tobacco Epidemic, 2009: Implementing smoke-free environments World Health Organization, Geneva, 2009. [acessado 2010 ago] Disponível em: www.who.int/tobacco/mpower/en/

25. Organização Pan-Americana de Saúde (OPAS). São Paulo respira melhor: adoção de ambientes fechados livres de tabaco no maior estado brasileiro. Brasília 2010. [acessado 2010 ago]. Disponível em: http:/ /actbr.org.br/uploads/conteudo/422_ESTUDO_ DE_CASO_SP.pdf

26. Fumo em locais fechados - Brasil, Pesquisa de Opinião Pública, 2008. [acessado 2010 ago]. Disponível em: http://actbr.org.br/uploads/conteudo/ 105_Fumo-em-Locais-Fechados-Datafolha-2008. pdf

27. Opinião de funcionários de bares, restaurantes e casas noturnas sobre fumo em locais fechados, Pesquisa de opinião pública, 2009. [acessado em 2010 ago]. Disponível em: http://actbr.org.br/uploads/ conteudo/253_Opiniao-Funcionarios-Datafolha2009.pdf

Artigo apresentado em 13/09/2010

Aprovado em 06/12/2010

Versão final apresentada em 16/02/2011 